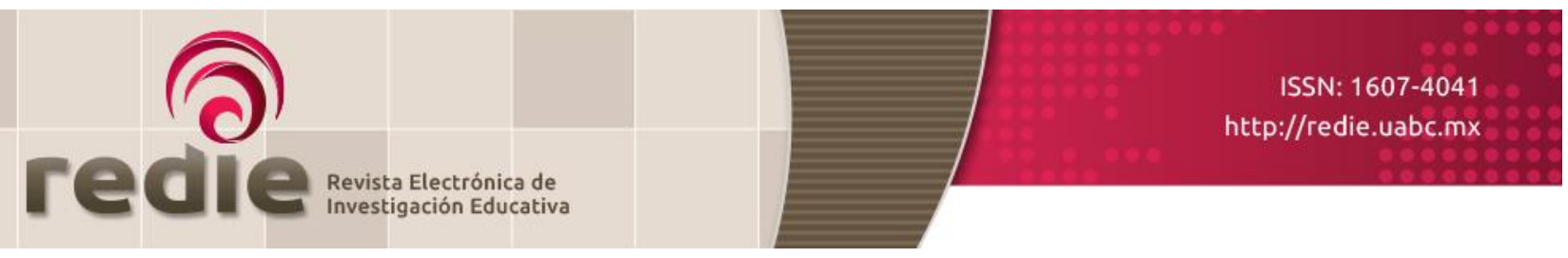

Vol. 19, Núm. 3, 2017

\title{
Aprendizaje basado en proyectos para el desarrollo de competencias matemáticas en Bachillerato
}

\section{Project-Based Learning for the Development of Mathematical Competencies in High School}

Gloria Flores-Fuentes (1) yoyis.Flores@gmail.com

Estela de Lourdes Juárez-Ruiz (2) ejuarez@ece.buap.mx

(1) Bachillerato General Oficial "Benito Juárez" de San Juan Raboso

(2) Benemérita Universidad Autónoma de Puebla

(Recibido: 21 de abril de 2014; Aceptado para su publicación: 3 de diciembre de 2015)

Cómo citar: Flores-Fuentes, G. y Juárez-Ruiz, E. L. (2017). Aprendizaje basado en proyectos para el desarrollo de competencias matemáticas en Bachillerato. Revista Electrónica de Investigación Educativa, 19(3), 71-91.

https://doi.org/10.24320/redie.2017.19.3.721

\section{Resumen}

Existe evidencia de que el aprendizaje basado en proyectos es una aproximación didáctica eficaz para formar competencias, desarrollar el pensamiento crítico y creativo y mejorar la motivación de los estudiantes. Este trabajo presenta los hallazgos de un caso de estudio en una escuela de nivel medio superior localizada en Puebla (México), en la que se implementa el aprendizaje basado en proyectos en el curso de Geometría y Trigonometría. Se hicieron ajustes en los enfoques al programa de la asignatura que establece la Reforma Integral de la Educación Media Superior (RIEMS), tanto en las actividades propuestas como en la forma de evaluación, y se diseñó una situación problemática a resolver por los estudiantes, la secuencia didáctica del proyecto, algunos materiales para las actividades y una matriz de desempeño para valorar el desarrollo de las competencias establecidas. Los resultados del estudio revelaron que los estudiantes experimentaron un aprendizaje más interesante y significativo de Geometría y Trigonometría y mejoraron su motivación para aprender Matemáticas.

Palabras clave: Proyectos escolares, competencias, educación media superior.

\section{Abstract}

There is evidence that project-based learning is an effective teaching approach to build competencies, develop critical and creative thinking and improve student motivation. This work presents the findings of a case study in a high school in Puebla (Mexico) that introduced project-based learning in a geometry and trigonometry course. Adjustments were made to the approaches in the syllabus set by the Comprehensive Reform of Higher Secondary Education (RIEMS, in Spanish), both in the activities proposed and the method of assessment. A problem situation was devised for the students to solve, and the didactic sequence of the project, material for activities and a performance matrix to assess competency development were designed. The study results show that students experienced more interesting and 
meaningful learning in geometry and trigonometry and were more motivated to learn mathematics.

Keywords: Student projects, competences, high school.

\section{Introducción}

La enseñanza y el aprendizaje de las Matemáticas, orientada científicamente por la didáctica presenta actualmente "redefiniciones y desafíos" (Artigue, 2004; D’Amore, Godino y Fandiño, 2008; Vanegas y Escobar, 2007, citados en García, Coronado y Montealegre, 2011) centrados en activar la construcción del conocimiento matemático por parte de los estudiantes en el complejo y dinámico proceso de la formación y el desarrollo de competencias matemáticas.

También existe una atención creciente en las herramientas de la actividad matemática y en los procesos de instrumentación asociados (Artigue, 2004). El profesor-facilitador de educación media superior en México, que desde el 2008 se ha involucrado en el proceso de aprendizaje-enseñanza-evaluación con el enfoque de competencias, se ha interesado en el diseño, desarrollo e instrumentación de estas herramientas del trabajo matemático en el contexto -situado, se diría- de los estudiantes, pues como afirma D'Amore (2014):

El trabajo didáctico que realiza el docente no consiste en trabajar sobre el conocimiento del sujeto que aprende, sino sobre las condiciones creadas por las situaciones puestas en acción en la situación de aprendizaje, teniendo presentes los esquemas y la adaptación. (p. 219)

También se ha preocupado por coadyuvar a mejorar la actitud de los estudiantes hacia el aprendizaje, pues "la competencia implica también un 'desear hacer', lo cual involucra hechos afectivos como volición y actitud" (D'Amore, 2008, p. 39). Por ello, en este trabajo se establecen las siguientes preguntas de investigación: ¿Cómo lograr que los estudiantes de educación media superior desarrollen competencias matemáticas y se encuentren motivados durante el proceso? ¿Cómo diseñar procesos de aprendizaje que guíen la labor del profesor-facilitador para lograrlo?

Desde la perspectiva de una visión compleja de la realidad, García et al. (2011) sitúan el concepto de competencias "en el complejo proceso de formación y desarrollo de un ser humano, en permanente actividad y con capacidades para acceder a nueva información y apropiarse de nuevo conocimiento" ( $p$. 61).

En la Reforma Integral de la Educación Media Superior (RIEMS) en México, las competencias disciplinares básicas son conceptualizadas como "los conocimientos, habilidades y actitudes asociados con las disciplinas en las que tradicionalmente se han organizado el saber y que todo bachiller debe adquirir" (RIEMS, 2008, p. 3). Se desarrollan en los campos disciplinares de Matemáticas, Ciencias Experimentales, Ciencias Sociales y Comunicación.

En el caso de las competencias matemáticas, el acuerdo 444 de la RIEMS, establece:

Buscan propiciar el desarrollo de la creatividad y el pensamiento lógico y crítico entre los estudiantes... [coadyuvan a] estructurar mejor sus ideas y razonamientos... deben poder razonar matemáticamente, y no simplemente responder ciertos tipos de problemas mediante la repetición de procedimientos establecidos. Esto implica el que puedan hacer las aplicaciones de esta disciplina más allá del salón de clases. (SEP, 2008, p. 6)

Rico y Lupiáñez (2008) señalan que la competencia matemática es simplemente saber matemáticas y hacer cosas con ellas.

Tobón (2010) afirma que la evaluación de las competencias desde la perspectiva de la valoración supera el tener criterios y evidencias; va más allá de la determinación del nivel de aprendizaje alcanzado por los estudiantes buscando con ello que la valoración sea una auténtica experiencia de aprendizaje y formación 
integral. Por su parte, Arreguín, Alfaro y Ramírez (2012) establecen que la evaluación de las competencias matemáticas supone conocer el grado de dominio de las mismas, y que para conocer dicho domino es necesaria la intervención del alumno ante una situación-problema que sea lo más cercana posible a una situación real.

En lo que respecta a los instrumentos usados para determinar el grado de dominio de las competencias, Tobón (2010) propone una matriz de valoración que puede ser analítica o sintética y su elección depende de los fines que se persigan. Este instrumento de valoración proporciona información tanto cualitativa como cuantitativa (Frola, 2009; Ruiz, 2009; Tobón, 2010).

El aprendizaje basado en proyectos ha sido considerado por algunos especialistas como la integración del aprendizaje basado en problemas y el aprendizaje basado en proyectos, por las similitudes que presentan estas aproximaciones didácticas y que se han denotado indistintamente por PBL (del inglés Problem-Based Learning y Proyect-Based Learning). (English y Kitsantas, 2013; Guerra y Kolmos, 2011). En lo sucesivo PBL denotará únicamente al aprendizaje basado en proyectos.

En el PBL los estudiantes realizan un proyecto en un tiempo determinado para resolver un problema real que organice y dirija sus actividades, y un producto o prototipo final que ofrece una solución a la problemática, todo ello a partir del desarrollo y aplicación de aprendizajes adquiridos y del uso efectivo de recursos (Díaz, 2005; Blumenfeld et al., 2005). No es una actividad complementaria al aprendizaje, sino su fundamento. La mayoría de los proyectos incluyen lectura, escritura y matemáticas. Pretende que los estudiantes asuman una mayor responsabilidad de su propio aprendizaje, así como aplicar en problemas reales, las habilidades y conocimientos adquiridos en su formación. Su intención es encaminarlos a situaciones que los lleven a rescatar, comprender y aplicar lo que aprenden como una herramienta para resolver problemas y realizar tareas.

\section{Metodología}

El objetivo del estudio fue desarrollar algunas de las competencias matemáticas establecidas por la RIEMS para el curso de Geometría y Trigonometría en un grupo de estudiantes de primer año de Bachillerato de la escuela donde fue realizada la intervención, a través de la realización de un proyecto contextualizado y mejorar sus actitudes hacia la matemática.

La investigación es un estudio de caso de un grupo en una escuela rural pequeña de reciente creación (o en formación) en la que trabaja una de las autoras del artículo.

La población estudiada fue un grupo de 32 estudiantes de segundo semestre (17 mujeres y 15 hombres con edades entre los 15 y 17 años) perteneciente al Bachillerato General Oficial "Benito Juárez" de la comunidad de San Juan Raboso, en el estado de Puebla (México), el cual cuenta con una población estudiantil de 120 alumnos, cuatro docentes y el director. San Juan Raboso es una comunidad semi-rural que presenta altos índices de migración, los habitantes se dedican principalmente al cultivo de la caña o viven de las remesas que les envían sus familiares. Los estudiantes que asisten al Bachillerato provienen tanto de esa comunidad como de comunidades rurales aledañas, lo que permite tener diversidad cultural y social. Algunos de los estudiantes son bilingües, dominan perfectamente el inglés y el español, ya que nacieron en Estados Unidos.

\subsection{Diseño del proyecto contextualizado}

Se seleccionaron las siguientes competencias matemáticas a desarrollar, que forman parte de las competencias disciplinares básicas del modelo de la RIEMS:

- Formula y resuelve problemas, aplicando diferentes enfoques.

- Argumenta la solución obtenida de un problema, con métodos numéricos, gráficos, analíticos o variacionales, mediante el lenguaje verbal, matemático y el uso de las Tecnologías de la Información y la Comunicación (TIC). 
Los contenidos seleccionados de la materia de Geometría y Trigonometría fueron:

- Congruencia y Semejanza de triángulos.

- Teorema de Pitágoras.

- Relaciones trigonométricas de un triángulo rectángulo.

- Pendiente.

Se eligieron estos temas porque además de ser un eslabón importante en la construcción de conocimientos para las asignaturas de Cálculo y Física, y en general una herramienta muy útil para la resolución de problemas de la prueba PLANEA (INEE, 2015), se deseaba observar el aumento de la complejidad en la actuación de los aprendices, desde la comprensión de conceptos hasta su aplicación en la resolución de un problema real de su entorno.

Se propuso que el diseño del proyecto contextualizado fuera realizado en trabajo colaborativo por los estudiantes, con una estructura de cinco etapas descrita a continuación.

Primera Etapa: De exploración. Los estudiantes exploran una situación problemática de su entorno, la cual se plantea de forma incompleta, como un problema mal estructurado, complejo y con múltiples soluciones (Jonassen, 1997). Se permite el libre abordaje de la misma. El que los estudiantes estudien el problema desde diversos enfoques -múltiples dimensiones- les permite conocer a fondo el problema, atrae su atención e interés y desarrolla su pensamiento crítico (Goodson, 2000).

Segunda Etapa: Identificación de un problema. Los estudiantes identifican un problema y proponen soluciones desde diversas perspectivas, las analizan y seleccionan una. Con esta actividad se promueve el desarrollo del pensamiento divergente y creativo, y la toma de decisiones (Goodson, 2000). Paralelamente a estas actividades, el facilitador trabaja en clase problemas guiados, aplicando conceptos y técnicas de los temas de estudio, desarrollando conocimientos y habilidades que servirán de soporte para el diseño y construcción de su prototipo. Collins (1997) llama a estas actividades, "actividades de andamiaje".

Tercera Etapa: De profundización. El facilitador establece un conjunto de condiciones a ser cumplidas en la solución propuesta: la utilización de los conceptos matemáticos, métodos y técnicas estudiados en clase. Esta nueva situación obliga a los estudiantes a analizar nuevamente su propuesta de solución para modificarla, completarla o ampliarla para cumplir con los nuevos requisitos. Es importante observar que estas condiciones específicas relacionadas con los temas de la asignatura no se dan a conocer al estudiante al principio del proyecto, debido a que el propósito de las primeras dos etapas es el libre abordaje del problema, el descubrimiento y la motivación que les permita involucrarse en su proyecto y fomentar su creatividad. Por otro lado, en la tercera etapa se pretende que el estudiante aplique los conocimientos estudiados en el aula y desarrolle sus habilidades de pensamiento crítico y creativo.

Cuarta etapa: De implementación. En esta etapa, los estudiantes desarrollan un prototipo donde implementan su propuesta de solución aplicando los conocimientos adquiridos.

Quinta etapa: De exposición de resultados y metacognición. Los estudiantes exponen su proyecto a la comunidad escolar. Al terminar esta actividad se realiza un análisis metacognitivo que les permite reflexionar sobre los aprendizajes adquiridos.

\subsection{Desarrollo de la propuesta de aprendizaje}

El proceso de aprendizaje se diseñó a través de una secuencia didáctica contextualizada que Tobón (2010) afirma "permite a los estudiantes un aprendizaje que contribuye al desarrollo de competencias" con actividades de inicio, desarrollo y cierre, como se muestra en la tabla I.

Tabla I. Secuencia didáctica contextualizada 


\begin{tabular}{ll}
\hline Situación problemática & Actividades de la secuencia didáctica \\
\hline El puente de San Juan & De inicio \\
Raboso, Puebla, México. & 1. Visita al puente. \\
\hline & De desarrollo \\
& En equipo colaborativo: \\
& 1. Observar la situación problemática e identificar un problema. \\
& 2. Establecer una propuesta de solución a la problemática detectada. \\
& Paralelamente a esta actividad, en clase: Resolución de problemas guiados aplicando \\
& conceptos, criterios, fórmulas y métodos de los temas seleccionados. \\
& 3. Proponer una solución al problema de su entorno, sujeta a las condiciones \\
& establecidas por el facilitador. \\
& 5. Elaborar el producto final con la implementación de la solución propuesta. \\
& 6. Elaborar un reporte escrito del proyecto, en el que se describan las herramientas \\
matemáticas usadas en la solución propuesta. & \\
De cierre & 1. Exposición del trabajo. \\
2. Actividad metacognitiva en el aula.
\end{tabular}

La situación problemática fue establecida por las autoras haciendo una analogía con una experiencia de aprendizaje-enseñanza realizada en la Benemérita Universidad Autónoma de Puebla con diversos grupos, tanto de estudiantes como de profesores, en los que se aplicó el método de aprendizaje basado en problemas en un puente peatonal situado a las afueras de la misma (Juárez, Lombardero y Flores, 2013). La transferencia se hizo considerando un puente de la comunidad de la siguiente manera.

El puente de San Juan Raboso: La carretera Internacional a Oaxaca atraviesa la comunidad de San Juan Raboso y, en algún punto tiene un puente que es usado para atravesar el caudal del río Atotonilco; el puente representa un riesgo para los habitantes del lugar y para los vehículos que transitan por él debido a diversas causas entre las que destacan:

- La carretera es de alta velocidad y presenta un acotamiento en el puente.

- La carretera en la zona del puente no posee una extensión o banqueta para el paso de peatones y ciclistas.

- No existe un puente peatonal en esa zona que permita cruzar sin peligro.

- En temporada de lluvias el río se desborda, inunda el puente y provoca problemas de circulación.

Los problemas guiados para profundizar en los contenidos temático se seleccionaron del trabajo de Montes de Oca et al. (s. f.), un ejemplo de ellos se muestra en el Anexo A y para su evaluación se utilizaron dos instrumentos (ver Anexo B).

Se determinó que el producto final -descrito en la tabla I- fuera una maqueta en la que los estudiantes implementaran la solución propuesta. Para la actividad metacognitiva de cierre se estableció realizar una reunión plenaria con la pregunta detonadora: ¿Qué aprendizajes obtuviste durante la realización del proyecto?

\subsection{Instrumentos para la recolección de datos}

Para valorar el nivel de dominio de las competencias se diseñó un mapa de aprendizaje y se establecieron los siguientes criterios (ver "Criterios para una evaluación formativa", en Rosales (2003), para una mejor comprensión del tema):

- Analiza el problema desde diversos puntos de vista.

- Reconoce los problemas que son consecuencia del mal diseño del puente.

- Reconoce con precisión el proceso que lo llevó a la comprensión y propuesta de solución de la problemática del puente de San Juan Raboso.

- Explica con claridad y coherencia los criterios que le ayudaron a seleccionar una solución a la problemática.

- Toma en consideración las necesidades de la población al proponer la solución del problema. 
- El desarrollo de la propuesta contiene los elementos necesarios para su comprensión.

- Utiliza conceptos matemáticos acordes a la realización y construcción de su propuesta.

- Realiza una investigación adecuada para apoyar su propuesta.

Asimismo, se establecieron cinco niveles de logro de las competencias aplicando la taxonomía solo (Structure of the Observed Learning Outcome) establecida por Biggs y Collis (1982) para evaluar la complejidad del desempeño. Los cinco niveles de respuesta, con su respectivo descriptor y ponderación se diseñaron como se muestra en la tabla II: los primeros tres atienden a niveles crecientes de comprensión y aplicación de los conocimientos, los dos últimos involucran, además, habilidades de pensamiento crítico y creativo, como la argumentación y el desempeño autónomo.

Tabla II. Mapa de aprendizaje

\begin{tabular}{|c|c|c|}
\hline \multicolumn{3}{|c|}{ Proyecto: La problemática del puente de San Juan Raboso y sus posibles soluciones } \\
\hline Nivel & Descriptor & Ponderación \\
\hline Pre-formal & $\begin{array}{l}\text { Muestra un bosquejo bastante general de conceptos y problemas sin intentar } \\
\text { resolverlos. No organiza la información. Su acercamiento con las problemáticas es } \\
\text { superficial. Hace resumen de la información, hace mapas conceptuales. }\end{array}$ & 6 \\
\hline Receptivo & $\begin{array}{l}\text { Realiza problemas guiados y reconoce su utilidad en la solución de problemas } \\
\text { reales. Comprende problemáticas cotidianas y las expone mediante el uso del } \\
\text { lenguaje escrito. Comprende los conceptos y los aplica adecuadamente en la } \\
\text { solución de ejercicios. }\end{array}$ & 7 \\
\hline Resolutivo & $\begin{array}{l}\text { Resuelve ejercicios estableciendo relaciones entre ellos y reconoce su importancia } \\
\text { en la solución de problemáticas cotidianas. Analiza la problemática de acuerdo a } \\
\text { varios enfoques, usando conceptos matemáticos y recursos tecnológicos. } \\
\text { Argumenta la solución propuesta sólidamente. }\end{array}$ & 8 \\
\hline Autónomo & $\begin{array}{l}\text { Se desempeña autónomamente en la búsqueda de soluciones investigando por } \\
\text { cuenta propia lo que le interesa y resolviendo los contratiempos que se le presentan } \\
\text { tanto en la información como en la herramienta matemática que necesita para } \\
\text { resolver el problema. Argumenta la solución de la problemática evidenciando el uso } \\
\text { de las matemáticas y las TIc. }\end{array}$ & 9 \\
\hline Estratégico & $\begin{array}{l}\text { Propone soluciones de manera innovadora, creativa y emprendedora. Muestra un } \\
\text { fuerte compromiso social con su entorno. Adopta una postura crítica. Expone su } \\
\text { propuesta defendiendo su punto de vista con argumentos sólidos y con buenos } \\
\text { fundamentos matemáticos. }\end{array}$ & 10 \\
\hline
\end{tabular}

Para estimar la motivación de los estudiantes durante la realización del proyecto se diseñó una escala de valoración de actitudes, que se presenta en la siguiente sección.

Para la actividad metacognitiva de cierre se utilizó la técnica de observación directa y como instrumentos de apoyo y evaluación, la bitácora. La observación fue realizada por la facilitadora del curso durante todo el tiempo de realización del proyecto en los tres ambientes de aprendizaje utilizados: el puente peatonal de San Juan Raboso, el aula de clases, y la comunidad de la escuela durante las exposiciones del trabajo.

\section{Resultados}

\subsection{En el desarrollo del proyecto}

Se observó una buena participación de todos los estudiantes a lo largo del desarrollo del proyecto, resaltó un par de equipos que planificó desde el inicio el diseño de su maqueta (producto final del proyecto) elaborando dibujos acerca de lo que querían desarrollar para resolver la problemática planteada (los equipos realizaron dos tipos de maqueta: la ampliación del puente existente o el diseño de un puente completamente nuevo). 
Un caso especial fue el de una estudiante que prefirió trabajar individualmente; culminando el proyecto pero presentando dificultades para resolver los problemas guiados y desarrollar su prototipo. El resto de los estudiantes trabajó activamente y los avances solicitados por el facilitador fueron entregados a tiempo.

Para poder diseñar su maqueta, un equipo realizó una entrevista a los habitantes cercanos al puente. Algunos de los problemas mencionados fueron: la acotación del puente, el encharcamiento de agua en época de lluvia y la necesidad de un muro de contención, ya que en ocasiones los carros se impactan en las casas que están a la orilla de la carretera. Otro equipo tomó fotografías y puntualizó un problema de contaminación por basura en el río. El resto de los equipos se limitó a visitar el lugar de la problemática y hacer los avances necesarios.

Conforme los estudiantes resolvían los problemas guiados descubrieron la forma en que estos les ayudarían a construir su prototipo. En el caso del problema guiado "Semejanza de triángulos", lograron comprender la diferencia fundamental entre congruencia y semejanza, ya que para realizar su maqueta dos equipos consideraron la construcción de triángulos semejantes a los que habían observado en un puente de la ciudad aledaña (Izúcar de Matamoros, Puebla).

El problema guiado "Teorema de Pitágoras" ayudó a los equipos que usaron triángulos rectángulos en la construcción de su maqueta, usaron dicho concepto para calcular la longitud de la hipotenusa de los triángulos de un puente que usaron como guía.

Los problemas guiados acerca de las "Relaciones trigonométricas de un triángulo rectángulo y pendiente" no habían sido considerados en la propuesta inicial sino integrados durante el desarrollo del proyecto, ya que un equipo consideró importantes dichos conocimientos para su prototipo al incorporar y aplicar el concepto de pendiente para construir rampas para el acceso a discapacitados y ciclistas. A pesar de que no todos los equipos consideraron los mismos temas para la construcción de su prototipo, todos cumplieron con la resolución de los problemas guiados.

Para la exposición de proyectos a la comunidad de la escuela, los estudiantes realizaron una presentación en Power Point o en rotafolio. En el Anexo C se muestra un ejemplo de cada uno de ellos.

\subsection{En el nivel de desempeño de las competencias}

Al inicio del proyecto se realizó una valoración pre-test para ubicar el nivel de desempeño de las competencias de los estudiantes usando el mapa de aprendizaje descrito en la tabla II. De acuerdo con lo observado, los resultados mostraron que el $63 \%$ de los estudiantes se encontraba en el nivel pre-formal y el $36 \%$ restante apenas en el receptivo. Los aprendices se limitaron a realizar las tareas solicitadas, no revelaron autonomía ni iniciativa, se mostraron limitados para realizar un análisis más profundo, fueron totalmente dependientes de los apuntes y no consultaron otras fuentes de información. Cumplieron con la entrega de los ejercicios, resúmenes y mapas solicitados, algunos buscaron la ayuda del facilitador, pero sin mostrar motivación para hacer algo más de lo que se les solicitó.

Al finalizar el proyecto se valoró nuevamente a los aprendices con el mapa de aprendizaje observando que el $90 \%$ se encontraba en el nivel estratégico y el $10 \%$ restante en el nivel resolutivo, indicando un gran avance en el desempeño de los estudiantes.

\subsection{En la motivación de los estudiantes}

La valoración de actitudes al inicio del proyecto (ver tabla III) mostró que las frecuencias relativas se distribuyeron entre todos los indicadores, siendo las más altas en los indicadores "a veces" y "nunca", que muestran poco interés, como fueron: consulta frecuentemente, hace más de lo que se le pide, organiza y lidera al equipo y se esfuerza por conseguir el logro. En el indicador Ayuda a sus compañeros la frecuencia relativa en "siempre" fue del 37.5\%, lo cual reveló que había buena disposición de un pequeño grupo de estudiantes para el trabajo colaborativo. 
Tabla III. Frecuencias relativas de la valoración de actitudes al inicio del proyecto

\begin{tabular}{lcccc}
\cline { 2 - 5 } Indicadores & Nunca & Frecuencia (\%) \\
& A veces & $\begin{array}{c}\text { Casi } \\
\text { siempre }\end{array}$ & Siempre \\
\hline Muestra empeño al realizar la actividad & 21.8 & 31.2 & 28.1 & 18.7 \\
Toma la iniciativa en la realización de la actividad & 18.7 & 28.1 & 31.2 & 21.8 \\
Participa permanentemente & 21.8 & 28.1 & 31.2 & 18.7 \\
Consulta frecuentemente & 56.2 & 12.5 & 15.6 & 15.6 \\
Se esfuerza por superar sus errores & 12.5 & 34.3 & 37.5 & 15.6 \\
Hace más de lo que se le pide & 9.3 & 59.3 & 9.3 & 21.8 \\
Asume los errores con naturalidad & 34.3 & 21.8 & 25.0 & 18.7 \\
Organiza y lidera al equipo & 62.5 & 12.5 & 12.5 & 12.5 \\
Se esfuerza por conseguir el logro & 18.7 & 37.5 & 31.2 & 12.5 \\
Ayuda a sus compañeros & 31.2 & 12.5 & 18.7 & 37.5 \\
\hline
\end{tabular}

Otro hecho importante a observar fue que aproximadamente el $20 \%$ de estudiantes obtuvo el indicador "casi siempre" o "siempre" en 8 de los 10 criterios evaluados, mostrando este pequeño subgrupo una buena actitud hacia el aprendizaje.

Al aplicar nuevamente la valoración al final del proyecto (ver tabla IV) se observó una tendencia hacia las frecuencias "siempre" y "casi siempre", indicando una mejora importante en la motivación de los estudiantes hacia el aprendizaje, especialmente en los indicadores se esfuerza por superar sus errores, conseguir el logro propuesto y ayudar a sus compañeros, en los que las frecuencias relativas se repartieron en casi el 90\% entre los valores "siempre" y "casi siempre". Con frecuencias "siempre" o "casi siempre"; el $72.5 \%$ de los estudiantes mostró empeño al realizar la actividad y en promedio el $63 \%$ "siempre" o "casi siempre" tomó la iniciativa en la realización de actividades, participó e hizo lo que se le pidió.

Tabla IV. Frecuencias relativas de la valoración de actitudes al final del proyecto

\begin{tabular}{lcccc} 
& \multicolumn{3}{c}{ Frecuencia (\%) } \\
\cline { 3 - 5 } Indicadores & Nunca & A veces & $\begin{array}{c}\text { Casi } \\
\text { siempre }\end{array}$ & Siempre \\
\hline Muestra empeño al realizar la actividad & 0 & 27.5 & 24.1 & 48.4 \\
Toma la iniciativa en la realización de la actividad & 5.1 & 27.5 & 18.9 & 48.5 \\
Participa permanentemente & 6.8 & 27.5 & 17.2 & 48.5 \\
Consulta frecuentemente & 50.0 & 27.5 & 10.3 & 12.2 \\
Se esfuerza por superar sus errores & 0 & 1.7 & 79.3 & 19.0 \\
Hace más de lo que se le pide & 15.5 & 27.5 & 36.2 & 20.8 \\
Asume los errores con naturalidad & 5.1 & 25.8 & 56.8 & 12.3 \\
Organiza y lidera el equipo & 12.0 & 22.4 & 60.3 & 5.3 \\
Se esfuerza por conseguir el logro & 0 & 10.3 & 39.6 & 50.1 \\
Ayuda a sus compañeros & 1.7 & 8.6 & 17.2 & 72.5 \\
\hline
\end{tabular}

El análisis de correlación entre los indicadores mostró que antes del proyecto había una correlación negativa (-0.99) entre Muestra empeño al realizar la actividad y Ayuda a sus compañeros, pero al finalizar el proyecto cambió a una relación directa de 0.7 , lo cual indica que el desarrollo del proyecto contribuyó a mejorar el trabajo colaborativo entre los estudiantes. Las parejas Muestra empeño al realizar la actividad con Toma la iniciativa en la realización de la actividad y Muestra empeño al realizar la actividad con Participa permanentemente mantuvieron una correlación directa alta antes y después del proyecto (de 0.8 a 0.97 y de 0.9 a 0.96 , respectivamente).

Con respecto a la actividad metacognitiva al final del proyecto, a la pregunta detonadora ¿Qué aprendizajes obtuviste durante la realización del proyecto?, las respuestas más frecuentes fueron trabajar en equipo (93.3\%), investigar (80\%), haber aprendido la utilidad de los ángulos y pendientes (60\%), mencionó aprender la semejanza de figuras (93.3\%), y haber aprendido a utilizar software para hacer diapositivas (50\%). Los estudiantes se percataron también de que además de mencionar los aprendizajes 
que les parecieron muy importantes, habían obtenido otros no menos importantes que fueron indispensables para la realización del proyecto.

\subsection{Casos críticos}

En el grupo había ocho estudiantes que al inicio de la intervención mostraban apatía hacia el aprendizaje, no obstante, durante la realización del proyecto tres de ellos modificaron radicalmente su actitud hacia el trabajo, dos de ellos (que habían mostraron desinterés incluso en la elaboración de los problemas guiados) se desempeñaron excelentemente al desarrollar el proyecto con su equipo, liderando los trabajos o actividades, y en la exposición final fue su equipo el ganador para representar al Bachillerato en el "XV Encuentro Regional de Investigación y Enseñanza de la Física" realizado en la ciudad de Puebla ese mismo año. Los cinco estudiantes restantes aumentaron sólo un nivel en la valoración de desempeño.

\section{Discusión y conclusiones}

Los estudiantes desarrollaron el proyecto identificando libremente los problemas matemáticos a los que se enfrentaban derivados de la situación problemática en el puente de San Juan Raboso, lo que concuerda con Arreguín et al. (2012), y con lo planteado por Pajares, Sanz y Rico (2004) acerca de la dificultad que entraña traducir una situación problemática de un contexto real al mundo matemático. Se evidenció la ruptura del equilibrio que se genera como punto de partida para el aprendizaje.

Los resultados de la experiencia de aplicación del proyecto contextualizado a través de los niveles de desempeño logrados en el mapa de aprendizaje (ver tabla II), mostraron que los estudiantes desarrollaron aprendizajes de orden superior y desarrollaron habilidades de pensamiento crítico y creativo durante la creación de su producto final, en concordancia con las investigaciones de Thomas (2000), quien ha observado que el PBL incrementa en los estudiantes las habilidades de pensamiento crítico, resolución de problemas e impacta positivamente en su aprendizaje permanente. Liu (2003) y Doppelt (2003) también encontraron mayores habilidades de pensamiento cognitivo y creativo y motivación de los estudiantes en su proyecto, que los estudiantes de otros grupos. Bell (2010) observó que el PBL permite una mayor comprensión de temas, un aprendizaje profundo, un mayor nivel de lectura y motivación incremental para aprender. Los estudios de Boaler (1999) confirman que el PBL desarrolla ciertas habilidades de análisis de problemas matemáticos conceptuales y aplicados en los estudiantes que no son fáciles de obtener por medio de aprendizajes tradicionales.

Gultekin (2005) corrobora también que a través del PBL los estudiantes se convierten en mejores investigadores, solucionadores de problemas y pensadores de orden superior, como se pudo establecer en el estudio. Tovar-Gálvez y Cárdenas (2009) establecen que con el PBL la construcción del conocimiento está ligada a la acción y más específicamente a la interacción entre sujetos, quienes comparten experiencias que les permiten posibilitar situaciones de aprendizaje. Asimismo, desarrollan habilidades de colaboración y comunicación.

El proyecto permitió a los estudiantes vincularse con las problemáticas de su entorno, generar preocupación y empatía con los habitantes de su comunidad, analizar su realidad y proponer soluciones con responsabilidad. Con ello se confirma la tesis de Cenich y Santos (2005, p. 3): "El aprendizaje no es un proceso puramente interno, sino un constructo social mediado por el lenguaje utilizado en el discurso social, donde el contexto en el cual ocurre constituye el centro del aprendizaje mismo".

Más aún, dado que los estudiantes trabajaron colaborativamente de manera efectiva y mejoraron su expresión oral y escrita, los resultados incidieron también en el desarrollo de competencias genéricas (RIEMS, 2008) tales como: participa de manera efectiva en equipos diversos; y escucha, interpreta y emite mensajes pertinentes en distintos contextos mediante la utilización de medios, códigos y herramientas apropiadas.

Con respecto a las actitudes de los estudiantes hacia el aprendizaje, el proyecto contextualizado estimuló su compañerismo, entusiasmo y creatividad, determinando una modificación sustancial entre las 
actitudes negativas iniciales y las actitudes positivas finales en concordancia con los resultados de Ortega, Saura y Mínguez (1993), quienes afirman: "Se ha visto claramente, que el aprendizaje basado en proyectos influencia la motivación de los estudiantes positivamente". También Pintrinch y Schunk (2002) aseveran que el aprendizaje se ve directamente afectado porque proporciona motivación y, en muchos estudiantes que tienen interés y eficacia académica, proporciona una mayor disposición y compromiso cognitivo.

Durante la actividad metacognitiva los estudiantes evaluaron no sólo sus aprendizajes, sino también sus esfuerzos, motivaciones, intereses y niveles de productividad. Con esta actividad los estudiantes aprendieron a través del análisis de sus propios procesos cognitivos; reflexionaron sobre qué tan bien trabajaron en grupo, la manera en que contribuyeron al mismo y cómo negociaron, planificaron y escucharon a los demás, haciéndose conscientes de sus propias fortalezas y debilidades.

El aprendizaje basado en proyectos no sólo es una aproximación didáctica que puede guiar la labor del profesor para lograr aprendizajes significativos y de orden superior, sino construir competencias que incluyen el desarrollo de habilidades de pensamiento crítico y creativo, mejorar actitudes hacia el aprendizaje y es una metodología eficaz para el trabajo empírico. Muestra que es posible transferir propuestas de aprendizaje en diversos ambientes, tanto en urbanos como rurales a través de analogías.

\section{Referencias}

Arreguín, L. E., Alfaro, J. A. y Ramírez, M. S. (2012). Desarrollo de competencias matemáticas en secundaria usando la Técnica de Aprendizaje Orientado en Proyectos. Revista Iberoamericana sobre Calidad, Eficacia y Cambio en Educación, 10(4) 264-284.

Artigue, M. (2004). Problemas y desafíos en educación matemática: ¿Qué nos ofrece hoy la didáctica de la matemática para afrontarlos? Educación Matemática, 16(3), 5-28.

Bell, S. (2010), Project-Based Learning for the 21st century: skills for the future. The Clearing House, 83(2) 39-43.

Biggs, J. B. y Collis, K. F. (1982). Evaluating the quality of learning: the solo taxonomy. Nueva York: Academic Press.

Blumenfeld, P. C, Soloway E., Marx, R. W., Krajcik, J. S., Guzdial, M. y Palincsar, A. (1991). Motivating Project-Based Learning: sustaining the doing, supporting the learning. Educational Psychologist, 26(3-4), 369-398.

Boaler, J. (1999). Mathematics for the moment, or the millennium? What a british study has to say about teaching methods. Education Week, 17(29) 30-34.

Cenich, G. y Santos, G. (2005). Propuesta de aprendizaje basado en proyectos y trabajo colaborativo: experiencia de un curso en línea. Revista Electrónica de Investigación Educativa, 7(2). Recuperado de http://redie.uabc.mx/redie/article/view/133

Collins, A. (1997). El potencial de las tecnologías de la información para la educación. En C. Vizcarro y J. A. León (Eds.), Nuevas tecnologías para el aprendizaje. Madrid: Pirámide.

D’Amore, B. (2008). Competencias matemáticas. Bogotá, Colombia: Cooperativa Editorial Magisterio.

D’Amore, B. (2014). Reflexiones sobre algunos conceptos clave de la investigación en educación matemática: didáctica, concepto, competencia, esquema y situación. Paradigma, 35(2), 199-210.

Díaz, M. de M. (2005). Modalidades de enseñanza centradas en el desarrollo de competencias. Orientaciones para promover el cambio metodológico en el espacio europeo de educación superior. España: Universidad de Oviedo. 
Doppelt, Y. (2003). Implementation and assessment of PBL a flexible environment. International Journal of Technology and Design Education, 13(2), 55-72.

English, M. C. y Kitsantas, A. (2013). Supporting student self-regulated learning in problem- and projectbased learning. Interdisciplinary Journal of Problem-based Learning, 7(2), 128-150.

Frola, P. (2009). Competencias docentes para la evaluación (Diseño de reactivos para evaluar el aprendizaje). México: Trillas.

García, Q. B., Coronado, A. y Montealegre, Q. L. (2011). Formación y desarrollo de competencias matemáticas: una perspectiva teórica en la didáctica de las matemáticas. Revista Educación y Pedagogía, 23(59), 159-175.

Goodson L. A. (2000). Teaching and learning, strategies for complex thinking skills. Annual Proceedings of selected research and development papers, 1(2), 164-172.

Guerra, A. y Kolmos, A. (2011). Comparing problem based learning models: suggestions for their implementation. En J. Davies, E. de Graaff y A. Kolmos (Eds.), PBL across the disciplines: Research into best practice (pp. 3-16). Dinamarca: Aalborg Universitetsforlag.

Gultekin, M. (2005). The effect of project based learning on learning outcomes in the 5th grade social studies course in primary education. Educational Sciences: Theory and Practice, 5(2), 48-56.

Instituto Nacional para la Evaluación de la Educación. (2015). Recuperado de http://www.inee.edu.mx/images/stories/2015/boletines/prensa/DIPTICO PLANEA.pdf

Jonassen, D. H. (1997). Instructional design models for well-structured and ill-structured problem-solving learning outcomes. Educational Technology: Research and Development, 45(1), 65-94.

Juárez, E. L., Lombardero, J. A. y Flores F. G. (2013). L'apprentissage fondé sur des problemes: une structure pour la conception pédagogique et experiences de sa mise en oeuvre dans differents contextes [El aprendizaje basado en problemas: una estructura para el diseño pedagógico y las experiencias de su aplicación en diferentes contextos]. En G. Athanaze (Ed.), Conferences du master interculturel en innovation éducative prefalc Insa de Lyon (France), UTFPR (Brésil), BUAP (Mexique) (pp. 297-331). Verlag, Alemania: Editions Universitaires Européennes.

Liu, M. (2003). Enhancing learners' cognitive skills through multimedia design. Interactive Learning Environments, 11(1), 23-39.

Montes de Oca, O. J., Bañuelos, T. F., Calvillo, V. J., Torres, G. J. L., Carrasco, G. G., Romano, R. S. et al. (s. f.). Geometría y Trigonometría, libro del estudiante. Academia Institucional de Matemáticas del Nivel Medio Superior. México: Instituto Politécnico Nacional.

Ortega, R. P., Saura, S. J. P. y Mínguez, V. R. (1993). La formación de actitudes positivas hacia el estudio de las ciencias experimentales. Revista de Educación, 301, 167-196.

Pajares, R., Sanz, A. y Rico, L. (2004). Aproximación a un modelo de evaluación: el proyecto PISA 2000. Madrid: Secretaría General Técnica.

Reforma Integral de Educación Media Superior. (2008). Recuperado de http://www.oei.es/pdfs/reforma educacion media mexico.pdf

Rico, R. L. y Lupiáñez, J. L. (2008). Competencias Matemáticas desde una perspectiva curricular. Madrid: Alianza Editorial. 
Rosales, C. (2003). Criterios para una evaluación formativa. Madrid: Narcea.

Ruíz, M. (2009b). Cómo evaluar el dominio de competencias. México: Trillas.

Secretaría de Educación Pública. (2008). Acuerdo número 444. Recuperado de https://www.sep.gob.mx/work/models/sep1/Resource/7aa2c3ff-aab8-479f-ad93db49d0a1108a/a444.pdf

Tobón, S. S. (2010). Proyectos formativos: metodología para el desarrollo y evaluación de las competencias. México: Book Mart.

Tovar-Gálvez, J. y Cárdenas, N. (2009). Perspectivas en enseñanza-aprendizaje: formación en competencias y metacognición a través de proyectos. Revista Espíritu Científico en Acción, 5(10), 22-34, Recuperado de http://www.educacionbc.edu.mx/departamentos/investigacion/publicaciones/ espirituaccion/Archivos/10/A.pdf

Thomas, J. W. (2000). A review of research on project-based learning. Prepared for the autodesk foundation. Recuperado de http://www.bie.org/research/study/review of project based learning 2000 


\section{Anexo A}

Problema de Tales de Mileto para el tema de Congruencia y Semejanza de triángulos

Tales de Mileto es considerado uno de los siete sabios de Grecia. En una ocasión, un sacerdote egipcio le preguntó cuál podría ser la altura de la pirámide del rey Keops. Tales de Mileto reflexionó y a continuación contestó que no se conformaba con calcular a "ojo", sino que la mediría sin la ayuda de ningún instrumento. Se recostó sobre la arena y determinó la longitud de su propio cuerpo. Los sacerdotes le preguntaron qué era lo que estaba ideando y él les contestó: "Me pondré simplemente en el extremo de esta línea que mide la longitud de mi cuerpo y esperaré hasta que mi sombra sea igual de larga. En ese instante la sombra de la pirámide de vuestro venerado Keops también ha de medir tantos pasos como la altura de la pirámide".

El sacerdote, desconcertado ante la extrema sencillez de la solución se preguntó si acaso no había algún error, algún sofisma, pero Tales de Mileto añadió: "Pero si queréis que os mida esta altura a cualquier hora, clavaré en la arena mi bastón. ¿Veis?, por ejemplo ahora su sombra es aproximadamente la mitad de su longitud; por lo tanto, en este momento también la sombra de la pirámide mide más o menos la mitad de su altura. Ahora estáis en disposición de medirla con toda exactitud: os bastará comparar la longitud del bastón con la de su sombra para encontrar, mediante división o multiplicación de la sombra de la pirámide, la altura de ésta".

\section{Cuestionario:}

1) ¿Cuál es la idea fundamental del texto? Ponle un título que resuma dicha idea.

2) Explica con tus propias palabras cómo midió Tales de Mileto la altura de la pirámide de Keops.

3) Realiza un dibujo utilizando sólo figuras geométricas que exprese la idea.

4) Este descubrimiento de Tales de Mileto es el punto de partida de la teoría de la semejanza. Explica qué son figuras semejantes. ¿Es lo mismo semejanza que igualdad? Anota todas las diferencias que encuentres.

5) Describe las características de una pirámide en términos geométricos. 


\section{Anexo B}

Instrumentos de evaluación del Problema de Tales de Mileto

\begin{tabular}{|c|c|c|c|c|c|c|c|}
\hline \multicolumn{8}{|c|}{ Escala de estimación de frecuencias } \\
\hline \multicolumn{2}{|c|}{ Estudiante: } & \multicolumn{6}{|c|}{ Asignatura: Geometría y Trigonometría } \\
\hline \multicolumn{8}{|c|}{$\begin{array}{l}\text { Competencia: Argumenta la solución obtenida de un problema, con métodos numéricos, gráficos, analíticos o } \\
\text { variacionales, mediante el lenguaje verbal, matemático y el uso de las tecnologías de la información y la } \\
\text { comunicación. }\end{array}$} \\
\hline \multicolumn{8}{|c|}{ Actividad: Resolución de problema guiado: Tales de Mileto } \\
\hline \multirow{3}{*}{\multicolumn{3}{|c|}{$\begin{array}{c}\text { Escalas y criterios } \\
\text { de } \\
\text { evaluación } \\
\text { Indicador }\end{array}$}} & \multicolumn{5}{|c|}{ Escala valorativa } \\
\hline & & & $\mathrm{A}$ & $\mathrm{B}$ & $\mathrm{C}$ & $\mathrm{D}$ & $\mathrm{E}$ \\
\hline & & & Muy Alta & Alta & Buena & Defic. & $\begin{array}{c}\text { No } \\
\text { Logro }\end{array}$ \\
\hline \multicolumn{8}{|c|}{$\begin{array}{l}\text { 1. Reconoce las construcciones geométricas que le } \\
\text { ayudan a resolver el problema. }\end{array}$} \\
\hline \multicolumn{8}{|c|}{$\begin{array}{l}\text { 2. Argumenta con claridad las diferencias entre } \\
\text { semejanza e igualdad. }\end{array}$} \\
\hline \\
\hline \multicolumn{8}{|c|}{$\begin{array}{l}\text { 4. Muestra disponibilidad y entusiasmo para realizar la } \\
\text { actividad. }\end{array}$} \\
\hline \multicolumn{8}{|c|}{$\begin{array}{l}\text { 5. Apoya a sus compañeros en la resolución del } \\
\text { problema. }\end{array}$} \\
\hline \multicolumn{3}{|c|}{ Evaluado por: } & \multicolumn{3}{|l|}{ Firma: } & \multicolumn{2}{|l|}{ Fecha: } \\
\hline \multicolumn{8}{|c|}{ Rúbrica de valoración } \\
\hline $\begin{array}{l}\text { Criterios de } \\
\text { evaluación }\end{array}$ & Pur & & & & $\begin{array}{l}\text { Puntuación } \\
\text { máxima }\end{array}$ & $\begin{array}{l}\text { Pu } \\
\text { ob } \\
\text { el }\end{array}$ & $\begin{array}{l}\text { Untuación } \\
\text { btenida por } \\
\text { estudiante }\end{array}$ \\
\hline Forma & \multicolumn{4}{|c|}{$\begin{array}{l}\text { La portada cuenta con los requisitos solicitados ( } 3 \text { puntos) } \\
\text { Las construcciones geométricas están trazadas con juego } \\
\text { geométrico, o en su defecto con algún software ( } 7 \text { puntos) } \\
\text { Cuenta con los dibujos necesarios para llevar a cabo la actividad } \\
\text { (6 puntos) } \\
\text { Responde todos y cada uno de los puntos solicitados ( } 4 \\
\text { puntos) }\end{array}$} & \multicolumn{2}{|l|}{20} & \\
\hline \multirow[t]{2}{*}{ Contenido } & \multicolumn{4}{|c|}{$\begin{array}{l}\text { Argumenta las respuestas al cuestionario del problema con } \\
\text { claridad (8 puntos) } \\
\text { Sabe cuáles figuras geométricas usar para solucionar el } \\
\text { problema (8 puntos) } \\
\text { Las respuestas al cuestionario contienen argumentes apoyados } \\
\text { de las construcciones geométricas ( } 8 \text { puntos) } \\
\text { Sabe la diferencia fundamental entre semejanza y congruencia } \\
\text { (8 puntos) } \\
\text { Explica los componentes de una pirámide (8 puntos) }\end{array}$} & \multicolumn{2}{|l|}{40} & \\
\hline & & & & & $\begin{array}{l}\text { Calificaciór } \\
\text { total: }\end{array}$ & & \\
\hline \multicolumn{8}{|c|}{ Observaciones: } \\
\hline
\end{tabular}




\section{Anexo C}

Presentaciones de los proyectos hechas por los estudiantes

Ejemplo en power point

Solución al Puente en SAN

JUAN RABOSO

- Armando Aguilar Ramírez

- Oscar Manuel Gálvez Muñoz

- Ilvan Cesar Ortega Flores

- Yessica Stephany Castilla Chavéz

- Maria Magdalena Calderon Sanguino

○ Geometria y Trigonometria

- Gloria Flores fuentes

Imagen C.1. Diapositiva 1

\section{INTRODUCCION}

- Un puente peatonal elevado a escala para que los peatonales puedan cruzar el río que atraviesa la carretera y así mismo también poder cruzar de un lado de la carretera al otro.

Imagen C.2. Diapositiva 2

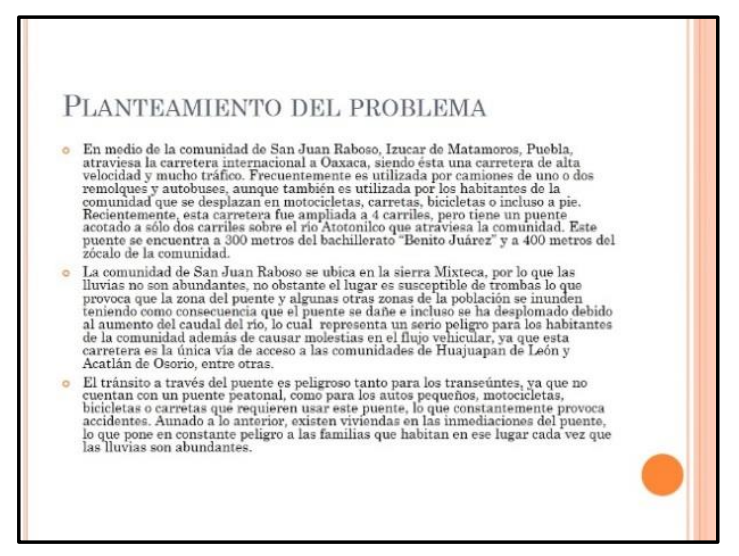

Imagen C.3. Diapositiva 3 


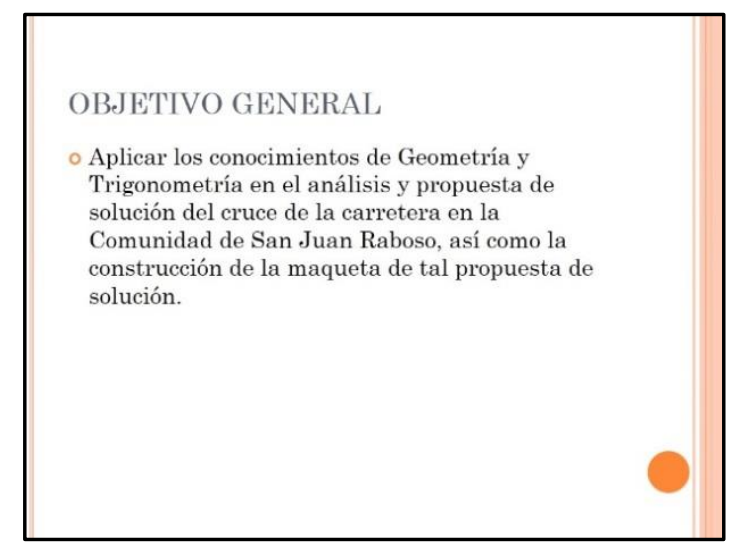

Imagen C.4. Diapositiva 4

\section{OBJETIVO ESPECÍFICOS}

○A) Describir la propuesta de la solución de la problemática del puente de San Juan Raboso

○B) Realizar una maqueta o un plano de dicha solución

oC) Describir que conocimientos de Geometría y Trigonometría utilizaron para resolver la problemática de solución de tu maqueta

oD) Presentar resultados

Imagen C.5. Diapositiva 5

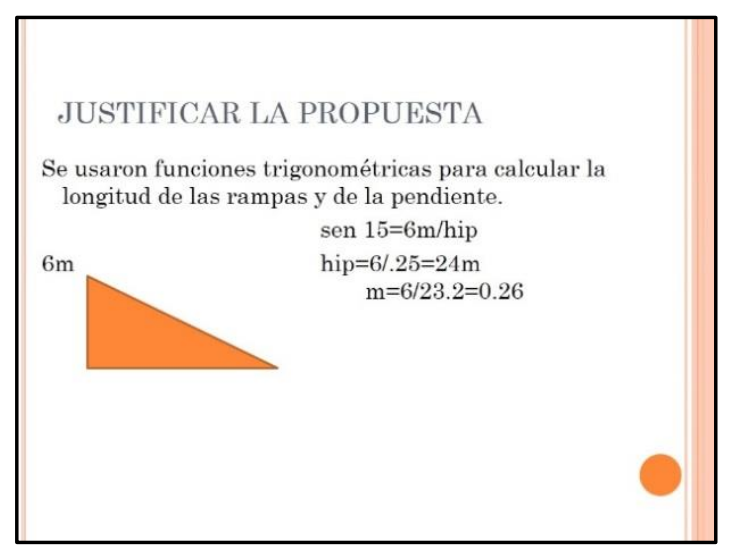

Imagen C.6. Diapositiva 6 


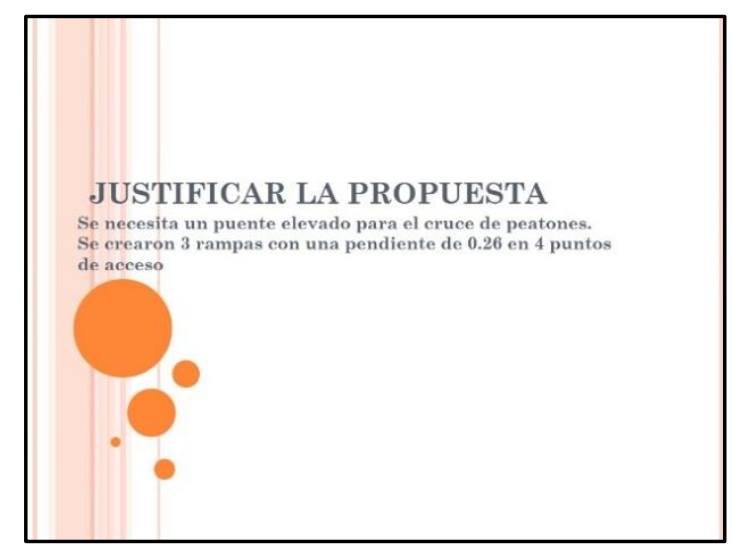

Imagen C.7. Diapositiva 7

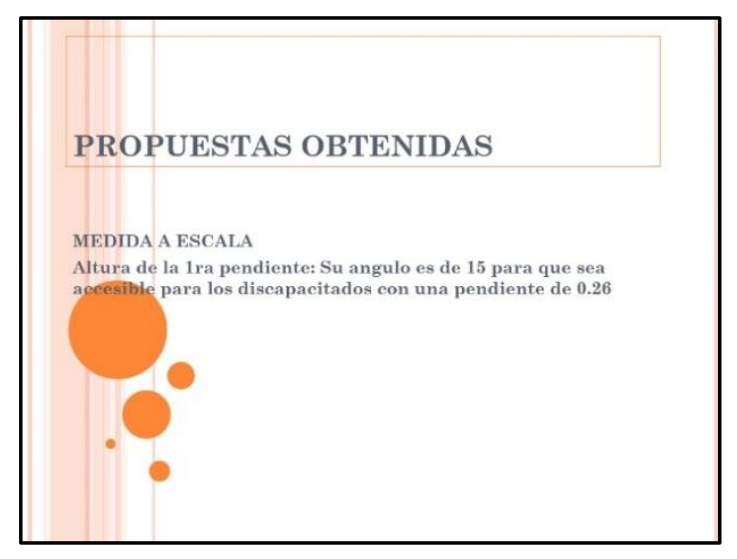

Imagen C.8. Diapositiva 8

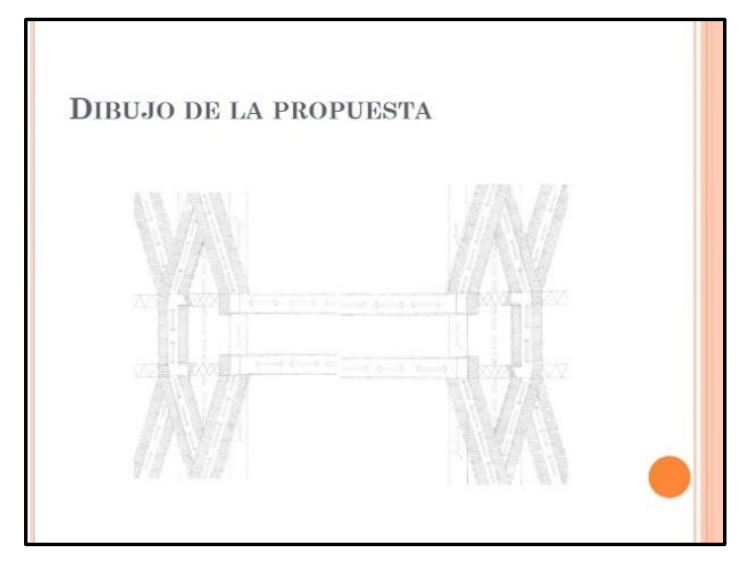

Imagen C.9. Diapositiva 9 


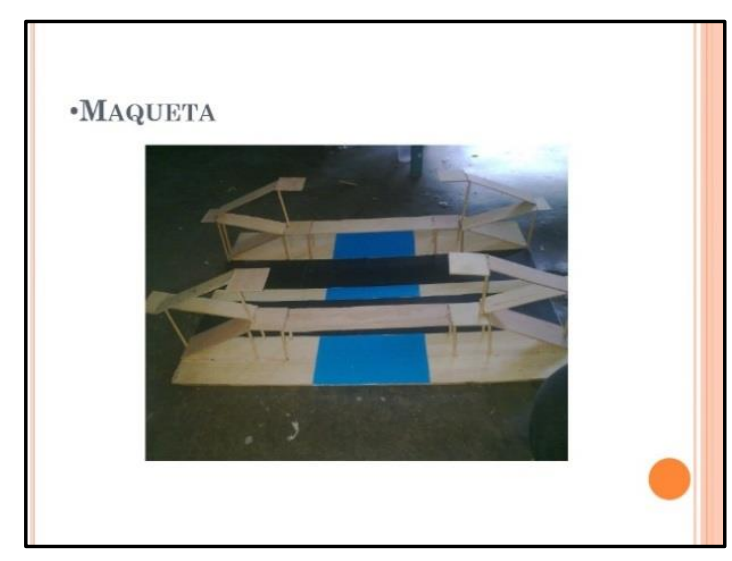

Imagen C.10. Diapositiva 10

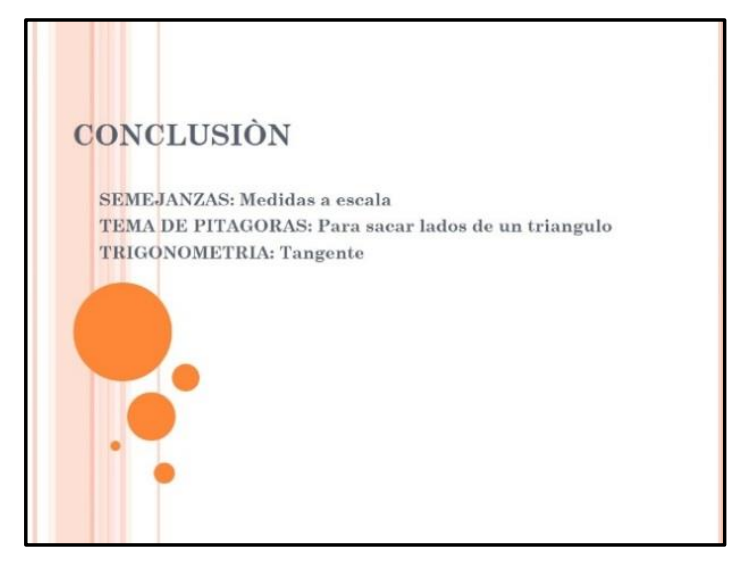

Imagen C.11. Diapositiva 11

\section{Presentación en rotafolio}

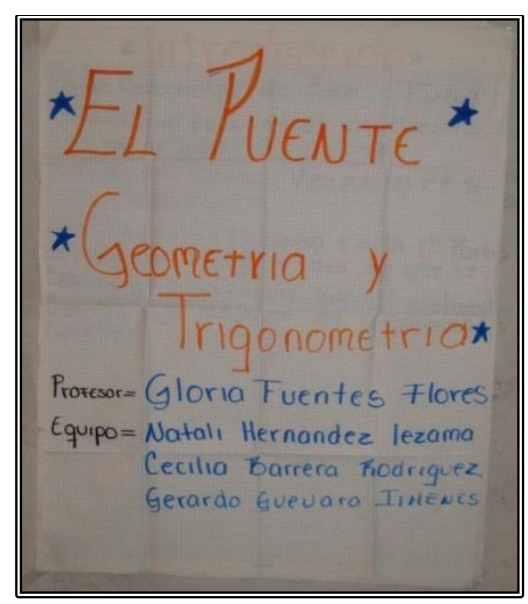

Imagen C.12. Rotafolio 1 


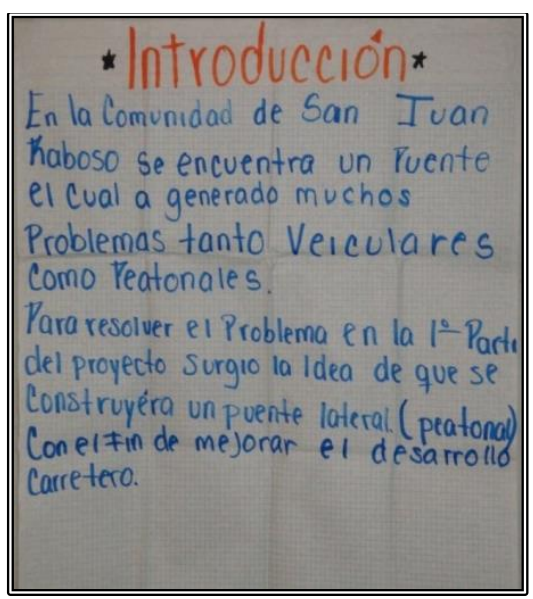

Imagen C.13. Rotafolio 2

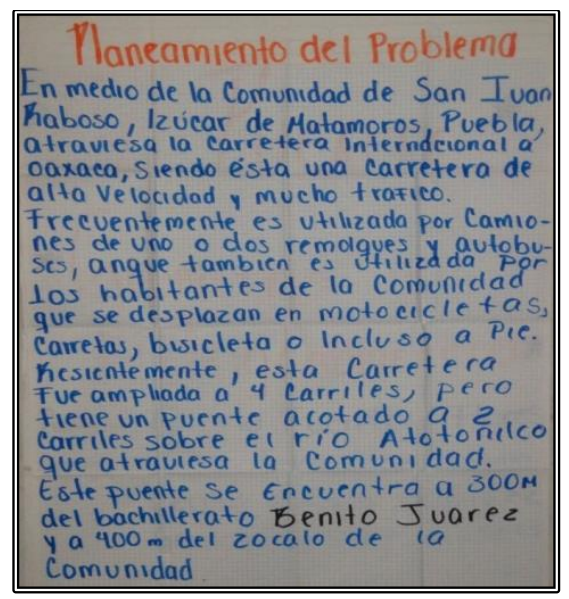

Imagen C.14. Rotafolio 3

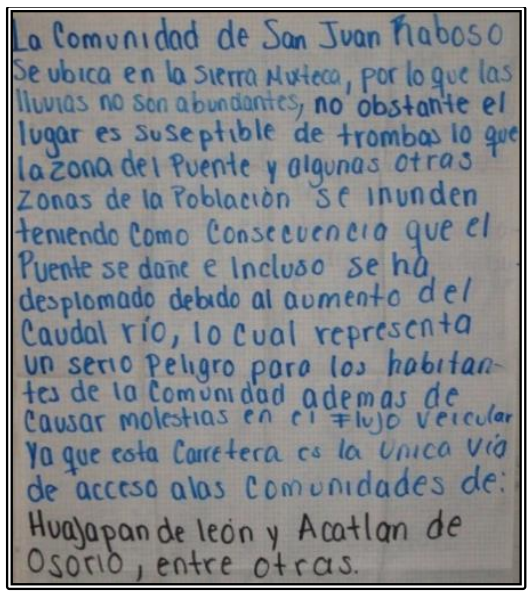

Imagen C.15. Rotafolio 4 


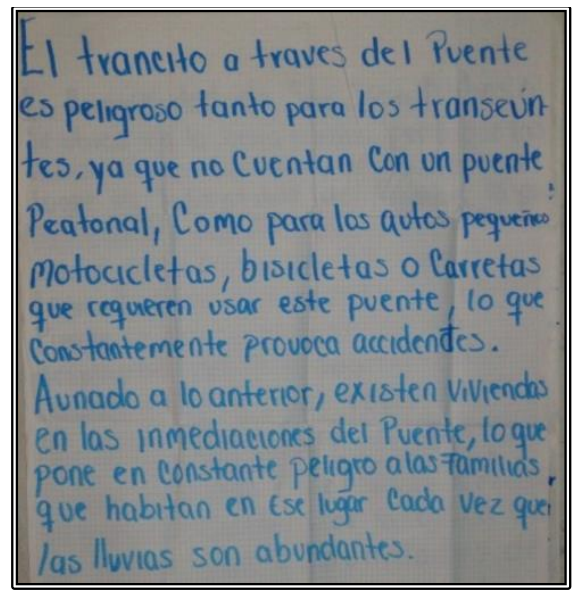

Imagen C.16. Rotafolio 5

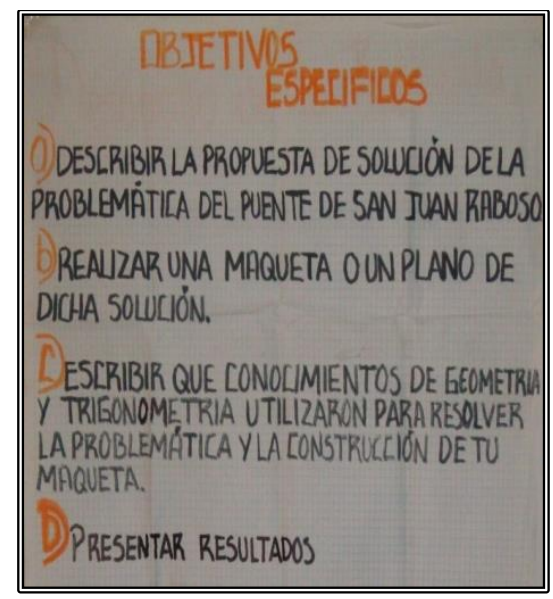

Imagen C.17. Rotafolio 6

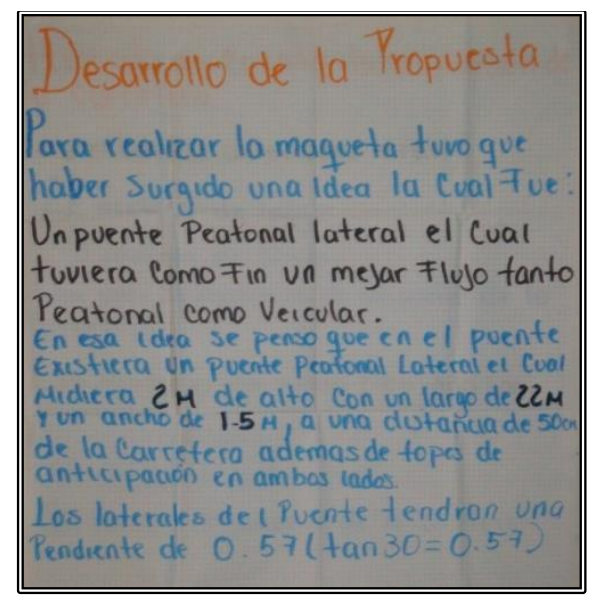

Imagen C.18. Rotafolio 7 


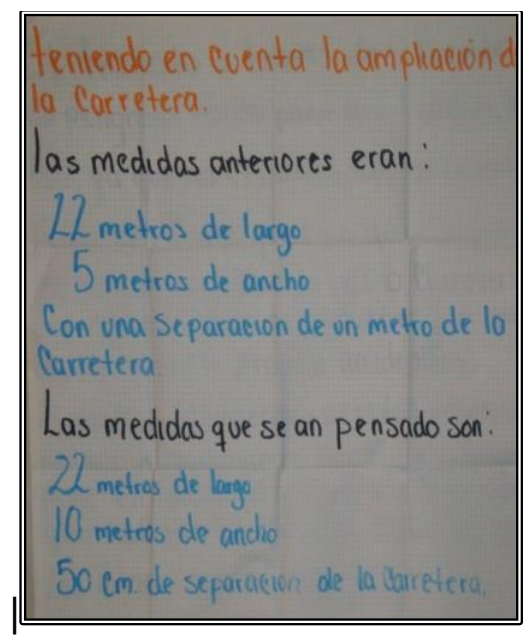

Imagen C.19. Rotafolio 8 